\title{
MDR TB Treatment of An Expectant Mother - A Case Report
}

\author{
A.Alaga*, M.A Fairous, M.K. Razul \\ Pulmonology Department, Hospital Sultanah Bahiyah, Alor Setar, Malaysia
}

Article Info

Article Notes

Received: February 12, 2020

Accepted: February 24, 2020

\section{${ }^{*}$ Correspondence:}

Dr. A.Alaga, Pulmonology Department, Hospital Sultanah

Bahiyah, Alor Setar, Malaysia; Email: arvindran_82@yahoo.com

C 2020 Alaga A. This article is distributed under the terms of the Creative Commons Attribution 4.0 International License.

\section{Introduction}

The burden of Multidrug - Resistant Tuberculosis (MDR-TB) is increasing both globally and locally. MDR -TB and its treatment pose a great risk to mother and fetus. The fear of fetal side-effects can sometimes impede proper treatment of the mother. There is little evidence to guide clinicians in the treatment of drug-resistant TB in pregnancy.

\section{Case Report}

We present a case of 32year old Malay lady known case of Type II Diabetes Mellitus (DM) presented with cough and loss of weight in April 2016. She has history of previous Pulmonary Tuberculosis (PTB) completed 6 months of antituberculosis treatment 2months ago.

Her chest radiograph showed left lower zone consolidation. Sputum for Acid Fast Bacilli (AFB) samples were positive, thus she was treated as relapse PTB smear positive. Gene Xpert of sputum was sent, and Rifampicin resistance was detected. Thus, she was started on Intramuscular (IM) Kanamycin, Levofloxacin, Ethionamide, Cycloserine, Ethambutol and Pyridoxine immediately. Her Mycobacterium Tuberculosis (MTB) culture sent during initiation of her treatment showed resistance to Isoniazide, Rifampicin, streptomycin and Pyrizinamide. The results of MTB culture were released around $2^{\text {nd }}$ month of her current treatment. She was on oral hypoglycemic agents for her DM.

She showed remarkable improvement with treatment. There was noticable weight gain and her cough resolved. Sputum AFB and MTB culture were negative after second month of therapy. Her chest radiograph showed improvement as well. She was then converted to maintenance therapy (for 12 months) in early 2017 after completing 240 doses ( 8 months) of intensive phase treatment, which consists of cycloserine, ethambutol, levofloxacin, ethionamide and pyridoxine.

However, during her $3^{\text {rd }}$ month of maintenance therapy, her urine pregnancy test was positive. Patient was urgently referred to Fetomaternal team whereby patient agreed to continue with pregnancy. She was monitored closely by both Pulmonology and Fetomaternal team. Her pregnancy was further complicated by uncontrolled diabetes. She was on subcutaneous insulin for her diabetes and on maintenance phase for MDR TB throughout her pregnancy. On 18th January 2018, she successfully delivered a baby girl. There was no evidence of perinatal transmission of tuberculosis to baby. Baby noted to have cleft palate, which was attributed to the uncontrolled diabetes in pregnancy. She completed her MDR TB 
treatment in February 2018. She didn't experience any adverse effects of MDR TB treatment. Currently baby and mother are well.

\section{Discussion}

Management of MDR TB in pregnancy is complex and pose many challenges. Insufficient safety data of treatment in pregnancy and potential teratogenicity effect carries a possibility to undertreat the mother. Most of the second line drugs are under category $C$ except aminoglycosides which are under category D. There have been several case studies published; some have shown no adverse perinatal outcomes, while others have recorded growth restriction and congenital defects

In a retrospective case study of 38 Peruvian pregnant women treated for MDR tuberculosis, $61 \%$ of them were cured, $13 \%$ died, and 5\% had experienced treatment failure. Eight (21\%) women experienced pregnancy complications, such as spontaneous abortion and vaginal bleeding. No infants displayed teratogenic effects. Among 5 South African pregnant women with MDR tuberculosis, 3 had HIV and 2 experienced adverse drug events (deafness and drug-induced hepatitis). The infants showed no evidence of teratogenicity

All female patients of childbearing age who are diagnosed with MDR TB should be tested for pregnancy and contraceptive advice should be given to sexually active patients. Pregnant patients should be started on treatment as soon as diagnosis made, however, it can be delayed till second trimester if the patient is stable with minimal disease.

The treatment should contain 3 or 4 second line anti$\mathrm{Tb}$ drugs plus pyrazinamide. Injectable agents should be avoided but capreomycin can be considered in life threatening situation. Treatment duration should be same as standard MDR -TB treatment

In our case, despite contraception advise was given, she was pregnant during maintenance phase of treatment. Potential teratogenic effect was explained, and termination of pregnancy option was given to patient and her husband. Prompt referral to Feto-maternal team was done.

Knowledge about the safety of bedaquiline and delamanid in pregnancy and while breastfeeding is sparse. WHO recommends that in pregnancy, a longer regimen be individualized to include components with a better safety profile. In our case, we used a longer regimen as above for total 20months of duration (8 months of intensive phase and 12 months of maintenance phase) to treat her MDR TB. She was treated successfully with no adverse drug effects noted on her and the baby.

\section{Conclusion}

Although pregnancy complicates the management of MDR-TB, continuing treatmentseem to outweigh theoretical risks to the mother and fetus. Favorable treatment and birth outcomes are possible with multidisciplinary approach and available drugs.

\section{References}

1. Palacios E, Dallman R, Muñoz M, et al. Drug-resistant tuberculosis and pregnancy: treatment outcomes of 38 cases in Lima, Peru. Clin Infect Dis. 2009; 48(10): 1413-9.

2. Tabarsi. Standardized second-line treatment of multidrug-resistant tuberculosis during pregnancy. Int J Tuberc Lung Dis. 2011; 15: 547-550.

3. WHO consolidated guidelines on drug-resistant tuberculosis treatment. 2019.

4. Multidrug-resistant tuberculosis in pregnant women Treatment and birth outcomes Andrii Dudnyk Olena Pavel'chuk European Respiratory Journal Sep 2016, 48. 